\title{
Periodicities Observed in Neutron Monitor Counting Rates Throughout Solar Cycles 20-24
}

\author{
Alejandro López Comazzi ${ }^{a, *}$ and Juan José Blanco Ávalos* \\ a Departamento de Física, Universidad de Alcalá, \\ Plaza de San Diego s/n, Alcalá de Henares, Spain \\ E-mail: alejandrofrancisco.l@edu.uah.es, juanjo.blanco@uah.es
}

Neutron monitor counting rates measurements from 1964 to 2019 corresponding with the Solar Cycles 20-24 have been analyzed in this work. A Global Neutron Monitor (GNM) was built in [4] for the years 2013-2018.

In this work, GNM is constructed over the last five Solar Cycles and is considered as a virtual representative station to characterize solar activity. Morlet wavelet analysis was applied to the GNM in order to determine periodicities.

The 27-day period and its second subharmonic (13-day period), related to solar synodic rotation, a periodicity between 45 and 84 days, the Rieger period ( 151 days) and nearly annual period have been detected in all SCs in both analyzed magnitudes. On a larger scales, 1.3-, 1.7-,11- and 22-year periods were obtained in GNM counting rates.

A temporal evolution of the periodicities obtained has been studied.

It is suggested that the same phenomenon produces the periodicities since their peaks are at similar times. This physical phenomenon could be a modulation effect related to solar rotation. We suggest the magnetic Rossby waves produce indirectly these periodicities in neutron monitor counting rates.

37th International Cosmic Ray Conference (ICRC 2021)

July 12th-23rd, 2021

Online-Berlin, German

\section{"Presenter}

*Thanks to project CTM2016-77325-C2-1-P funded by Ministerio de Economía y Competitividad and by the Eu ropean Regional Development Fund, FEDER.

$\$$ Thanks to MINECO - FPI 2017 Program cofinanced by the European Social Fund. 


\section{Introduction}

Galactic cosmic rays are charged particles, mainly atomic nuclei, with energies between $10^{6}$ and $10^{20} \mathrm{eV} /$ nucleon, originated in violent phenomena outside of our Solar System. The galactic cosmic ray flux that reaches Earth at low energies (energies bellow than several hundreds of $\mathrm{GeV} /$ nucleon) is deeply affected by the Solar Cycle whose average duration is 11 years [2]. This temporal variation of cosmic ray flux is due to the interaction of cosmic ray particles with the interplanetary magnetic field that is carried by solar wind.

Because of this, cosmic ray flux suffers a modulation process as they pass through the heliosphere, such that their flux is maximum during intervals of minimum solar activity and likewise their flux is minimum during intervals of maximum solar activity (i.e. when the number of sunspots is maximum). Thus, cosmic ray flux is anticorrelated to the solar activity, measured by sunspot number, with perhaps some time delay caused by irregularities in the Interplanetary Magnetic Field [1] .

The interaction with particles present in the Earth's atmosphere also affects cosmic rays. Due to such interaction cascades of secondary particles (such as protons, muons, neutrons and mesons) are created. Some of these secondary cosmic rays are detected by ground-based neutron monitors and muon telescopes.

In the year 1964, Hugh Carmichel developed a neutron monitor with a statistical accuracy of $0.1 \%$, called NM64. Similar instruments along different locations on the Earth have been developed subsequently. Most of them share their measurements in the Neutron Monitor Data Base (NMDB) currently. To study the solar activity through NMs is necessary to use multiple stations located in different geomagnetic positions. The reason for this is that the position of NMs with respect to the Earth's magnetic field determines the minimum energy of cosmic ray particles to generate counts on a given station. Nevertheless, a single neutron monitor representing a global behaviour of the neutron monitor network can be useful for some studies. [4] defined a neutron monitor representative of the neutron monitor network during the period 2013-2018. This detector is named as Global Neutron Monitor (GNM).

The study of periodicities in neutron monitor counting rates has been a widely studied topic. Some of the periodicities that stand out from the different studies are described bellow.

The cosmic ray flux shows a periodic modulation of $\sim 27$ days, associated to the solar synodic rotation and related with the coronal holes and co-rotating interaction regions [3].

Other well-known period is the Rieger-type period ( $\sim 151$ days). This period is unstable and appears around solar maximum coinciding with the change of polarity of the Sun. [5] and [6] have reported on a mid-term variation of 1.7 years in the neutron monitor counting rates. This period is related to emergence and transport phenomena of magnetic flux and is a multiple of the Rieger period.

The 11-year period, which is the most prominent periodicity recorded in neutron monitor counting rates, is related to the inversion of the solar global magnetic field. 
For this work, we establish the following operative classification: short-term periodicities refer to periods from 2 to about 30 days, mid-term periodicities are those periods found in the range between 30 days and 2 years and long-term periodicities refers periods greater than 2 years.

This paper examines the short-, mid- and long-term periodicities during the period 1964-2019 in GNM using the wavelet technique. GNM is a virtual station whose counting rate have been obtained by averaging of the counting rates, in typified units, of all selected neutron monitors for a particular SC.

\section{Results}

The Global Neutron Monitor (GNM) defined above has been built with the goal to obtain a representative measurement of global neutron monitor network along 32 years and fully comparable along five solar cycles. The errors in the periodicities have been calculated by approximating the peak to a Gaussian function, then the error is given by half the width at half the height of the Gaussian in all cases.

Figure 1 (upper panel displays the GNM counting rate from 1 October 1964 to 31 January 2019. Every SC is plotted in different colors to make easier the analysis.

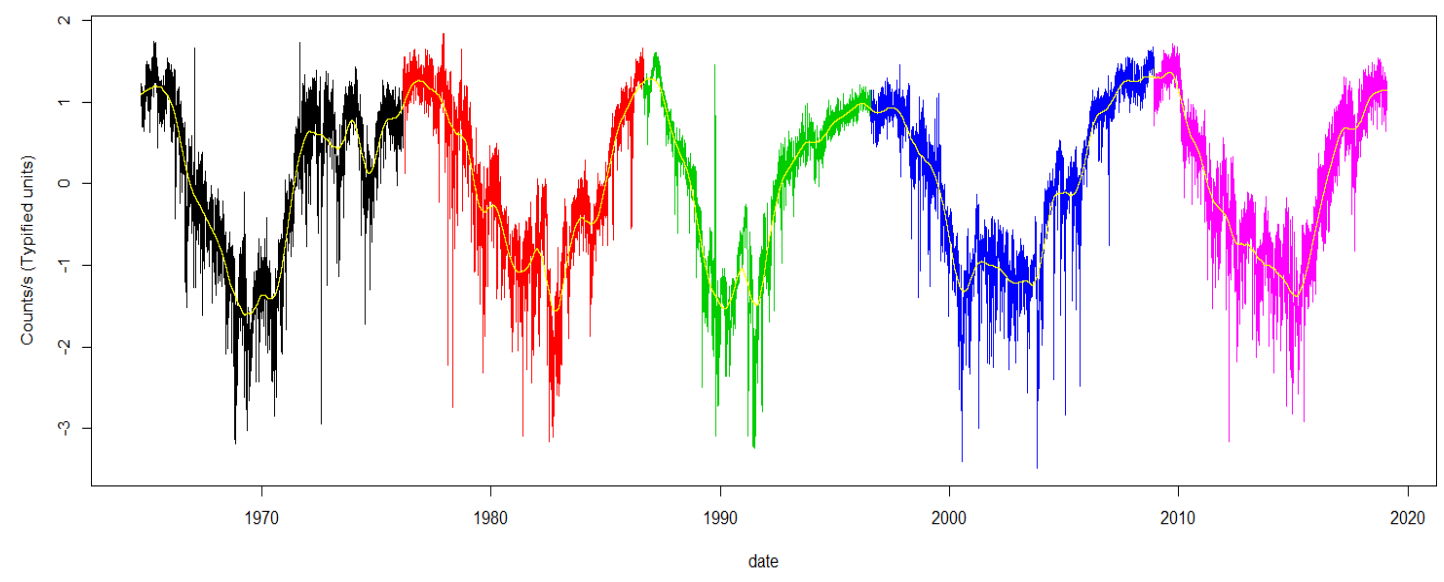

Figure 1: Global Neutron Monitor counting rates obtined by the average of counting rates of all selected neutron monitors (in typified units) in each Solar Cycle.

Wavelet Power Spectrum (WPS) of GNM in Solar Cycles (SCs) 20-24 is displayed separately in Figure 2. In general, periodicities are much more marked around the solar maximum in all SCs. The descending phase of the all SCs does not present a high spectral power for any periodicity except the 27-day period in SC24. In SC20, 27-day period appears along the entire interval with a strong spectral power, although it weakens at the beginning and end of this SC (i.e. in 1964-1966 and 1975-1976). This periodicity does not appear continuously but weakens in certain sections of the remaining SC.

The same is observed for the 14-day period that appears strongly throughout the SC20 weakening or disappearing in certain sections. The 14 and 27-day periods show the same 
behavior throughout the WPS applied to GNM in SC21, SC23 and SC24: they appear throughout the complete time series except in the beginning and the end of each cycle. The WPS of GNM in SC22 presents the same persistence of both periodicities but with a lower spectral power. Zones of high spectral power are observed between 32 and 128 days in certain parts of the ascending and decreasing phases of the SC20 and SC21.

However these zones are observed around the solar maximum in the SCs 22-24, disappearing in the rest of the interval. This last observation is especially evident in SC22. Around the solar maximum appears a very strongly marked period around the 189 days in SC20. In this interval (128-256 days) marked periodicities are observed around the solar maximum and at the beginning of the descending phase in all SCs.

Other zones of high spectral power have been observed around 365 days (nearly annual period) and 500 days appear around the solar maximum and are maintained during the descending phase. Although these periodicities overlapping with other minor or major periods during the descending phase. The Global Spectrum was calculated and the significant peaks have been collected in Table 1.

\begin{tabular}{lllrrrl}
\hline GNM & \multicolumn{7}{c}{ Period (days) } \\
\hline SC20 & $14 \pm 1$ & $27 \pm 2$ & $82 \pm 8$ & $189 \pm 17$ & $385 \pm 26$ & \\
\hline SC21 & $14 \pm 1$ & $27 \pm 3$ & $79 \pm 9$ & $153 \pm 16$ & $302 \pm 21$ & \\
\hline SC22 & $14 \pm 1$ & $27 \pm 3$ & $79 \pm 8$ & - & $214 \pm 17$ & $481 \pm 35$ \\
\hline SC23 & $14 \pm 1$ & $27 \pm 3$ & $63 \pm 6$ & $134 \pm 14$ & $228 \pm 20$ & $407 \pm 30$ \\
\hline SC24 & $14 \pm 1$ & $27 \pm 3$ & $46 \pm 7$ & $140 \pm 12$ & $311 \pm 23$ & $498 \pm 32$ \\
\hline
\end{tabular}

Table 1: Periodicities detected in GNM counting rates along the differents Solar Cycles

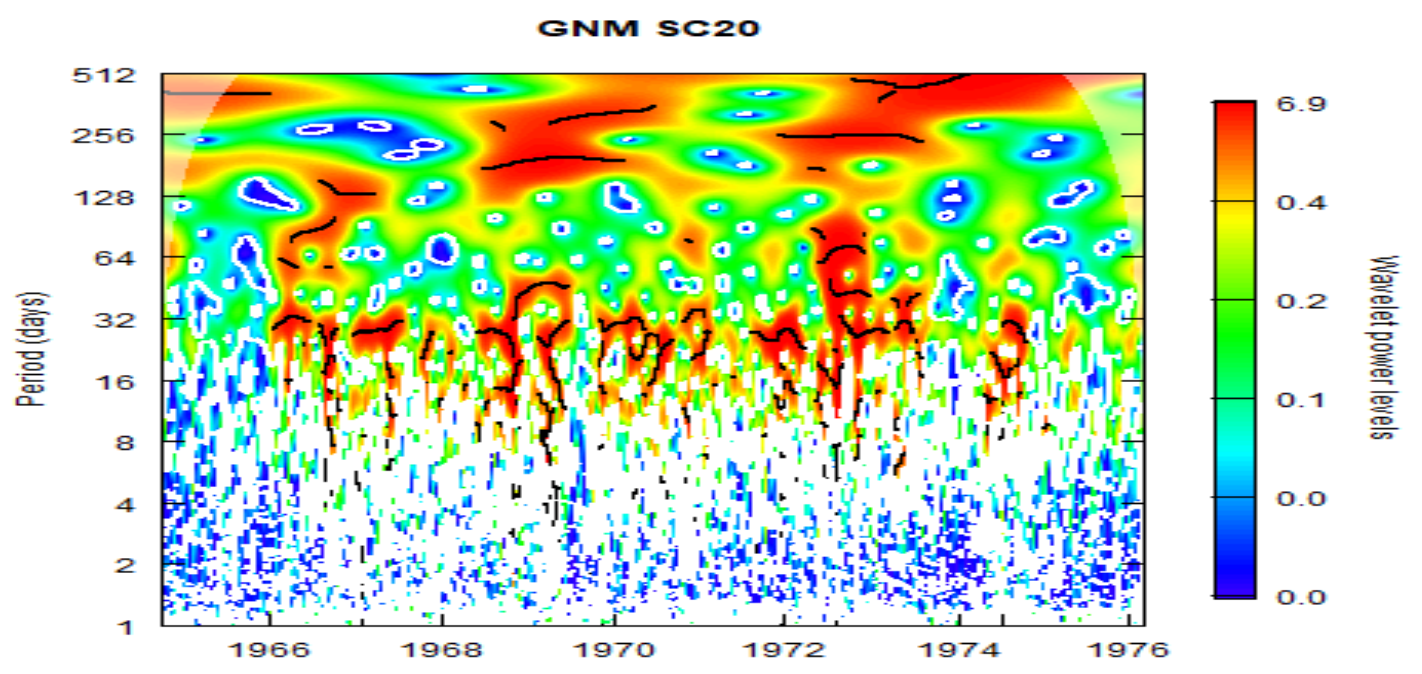



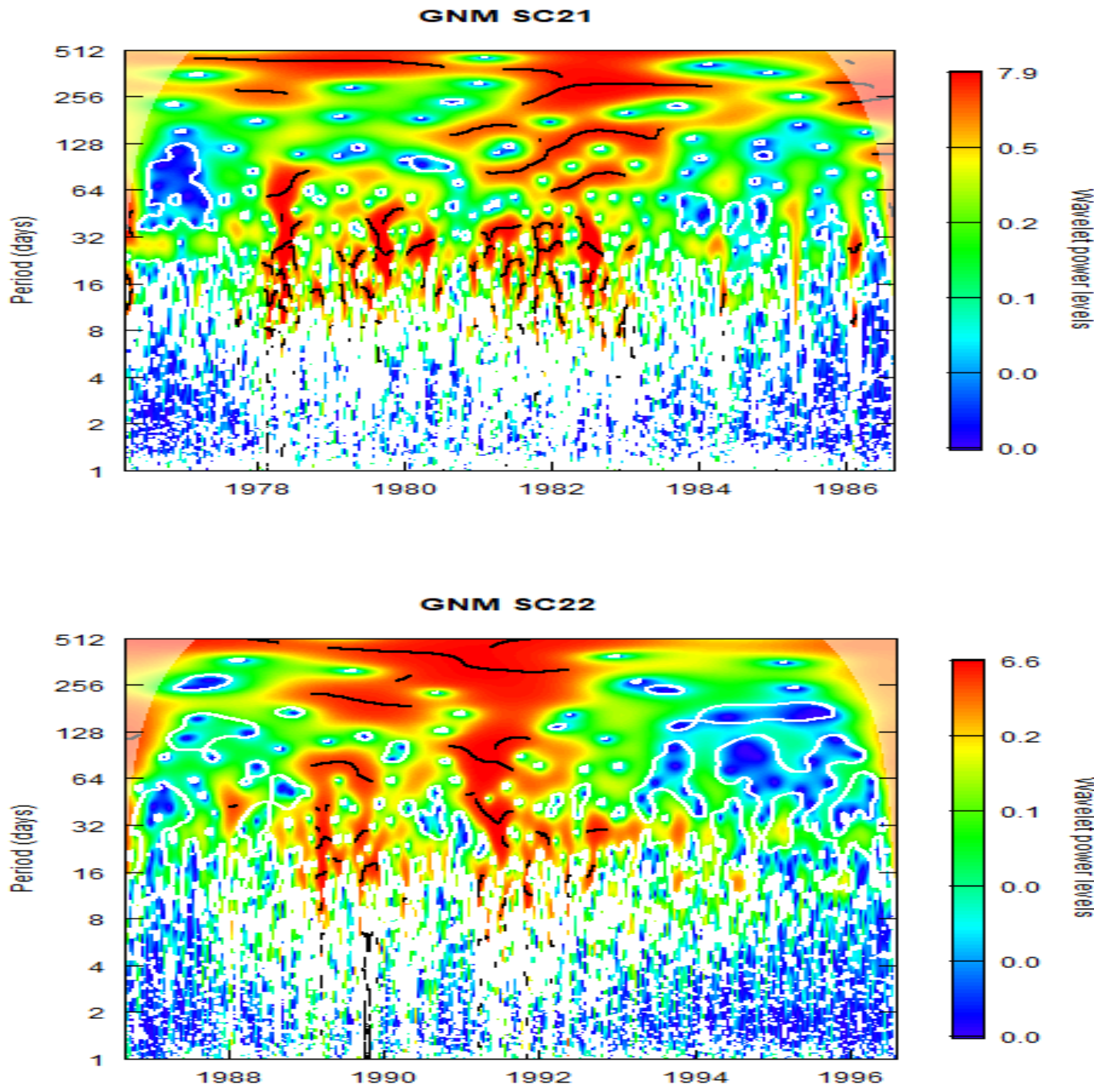

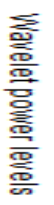

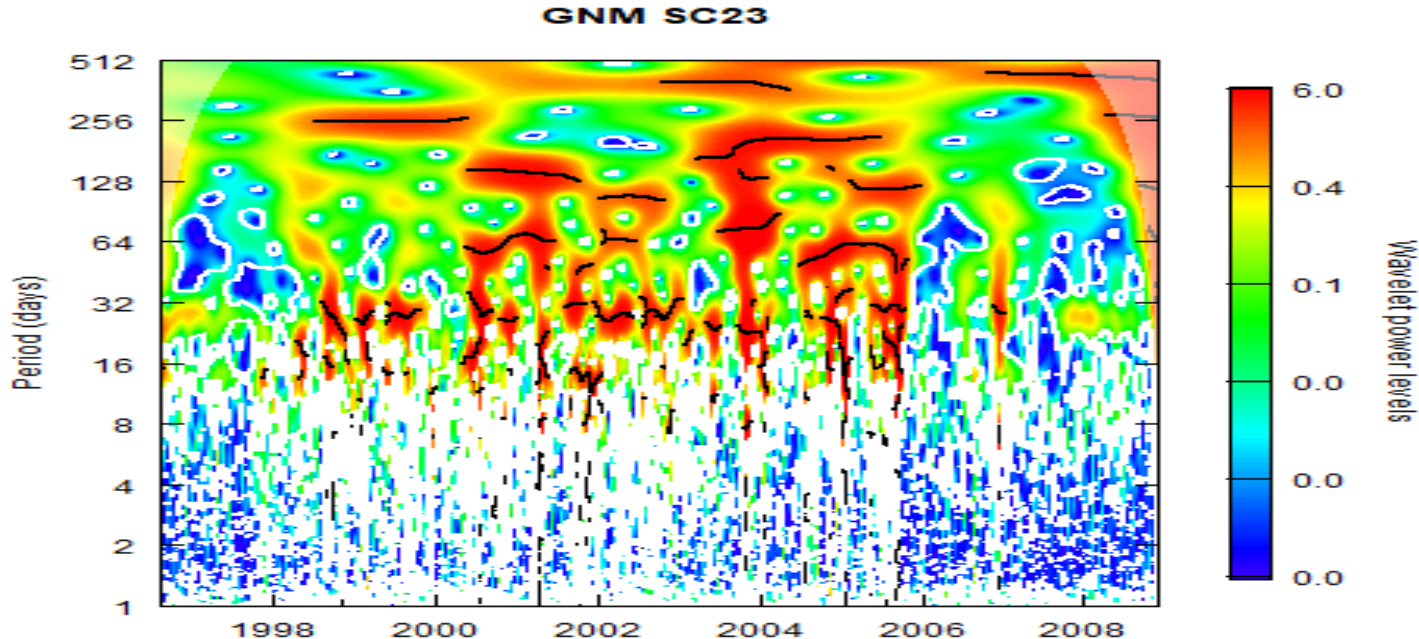




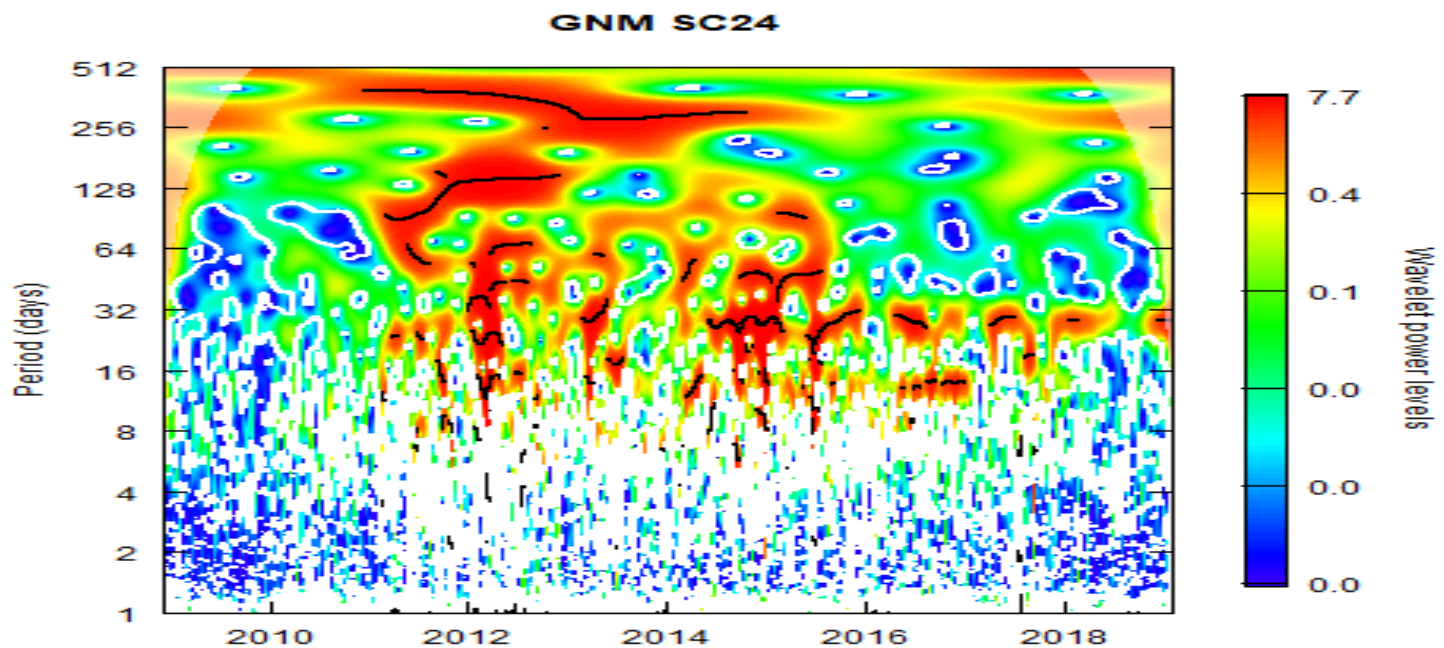

Figure 2: Wavelet Power Spectrum of GNM counting rates in each Solar Cycle.

Rossby waves have been proposed by recent studies to explain the periodicities observed in solar activity. Then, we explore whether there is a relationship between fast and slow Rossby magnetic waves and the short-, mid- and long-term periodicities obtained in the solar activity and GNM counting rates.

The Sun is a self-sustaining dynamo that converts convective motion and differential rotation within the Sun to electromagnetic energy. Therefore, the solar magnetic field is produced by the solar dynamo. The detailed mechanism of the solar dynamo is unknown and is currently under study. Current models place the solar dynamo in the tachocline, a transition zone between the convective region and the radiative zone. Magnetohydrodynamic "Shallow Water" model predicts the presence of waves in the tachocline.

Rossby waves are a consequence of the conservation law of angular momentum and their velocity is proportional to the rotational velocity of the system.

Fast Rossby magnetic waves have the characteristic that in the presence of a moderate magnetic field they become equatorially confined as the rotational speed of the system increases.

The angular frequency of fast Rossby waves according to [7] is given by

$$
\omega=\frac{-2 \Omega s}{\mathrm{n}(\mathrm{n}+1)}
$$

where $\Omega$ is the system rotational velocity, $\mathrm{n}$ and $\mathrm{s}$ are integer numbers defined as toroidal and poloidal wave number respectively $(\mathrm{n}=1,2,3, \ldots$ and $\mathrm{s}=0,1,2, \ldots, \mathrm{n})$. 
Considering $\Omega=2.65 \times 10^{-6} \mathrm{~s}^{-1}, \mathrm{~s}=1$ and $\mathrm{n}=1,2,3,4,5$, a set of waves with 27-, 82-, 165 , 274-, and 412-day period are obtained. These periods are similar to those obtained in GNM counting rates by wavelet analysis.

\section{Conclusions}

Global Neutron Monitor (GNM), has been calculated by averaging the counting rates in typified units of the different selected NMs in each SC following the criteria of [4]. The Morlet Wavelet Analysis is applied to the GNM counting rates in the interval 1964-2019, covering five SCs, and in each SC separately.

In short-term range, solar synodic rotation period ( 27-day) and its second sub-harmonic (13.5 day period) have been observed in all SCs both in GNM.

Rieger period ( 134-189 days), a period in 46-82 days and nearly annual period have been detected in GNM in all SCs in a mid-term range. Other periodicities obtained in GNM counting rates are in the ranges: 46-82, 214-302 and 385-498 days.

A temporal evolution of the periodicities . has been studied. It is suggested that a same phenomenon produces the periodicities since their most significant peaks coincide over time. This physical phenomenon could be a modulation effect related to solar rotation. We suggest the magnetic Rossby wave produce these periodicities in solar activity and also observed in neutron monitor counting rates.

The periodicities obtained by a Rossby wave model are coincident with the periods obtained in GNM counting rates. This model does not consider differential rotation.

It would be interesting in future studies to extend the model by adding differential rotation. This new model would increase the accuracy of the periodicities and likewise decrease the associated error in the periodicities.

\section{References}

[1] Forbush, S. E. 1958, J. Geophys. Res., 63, 651,doi: https://doi.org/10.1029/JZ063i004p00651

[2] Gnevyshev, M. 1967, SoPh, 1, 17,doi: https://doi.org/10.1007/BF00150306

[3] Grieder, P. 2001, Cosmic Rays at Earth by P.K.F. Grieder.Elsevier Science, 2001., -1

[4] López-Comazzi, A., \& Blanco, J. 2020, Short-term periodicities observed in neutron monitor counting rates. Solar Physics, 295,doi: https://doi.org/10.1007/s11207-020-01649-5

[5] Valdés-Galicia, J. F., Pérez-Enríquez, R., \& Otaola, J. A.1996, SoPh, 167, 409, doi: 10.1007/BF00146349

[6] Velasco Herrera, V., Pérez-Peraza, J., Soon, W., \&Márquez-Adame, J. 2018, New Astronomy, 60, 7 ,doi: https://doi.org/10.1016/j.newast.2017.09.007

[7] Zaqarashvili, T. V., Oliver, R., \& Ballester, J. L. 2009,ApJL, 691, L41, doi: 10.1088/0004637X/691/1/L41 